\title{
A new altimeter for Mars land shape observations utilizing the ionospheric sounder system onboard the Planet-B spacecraft
}

\author{
Hiroshi Oya and Takayuki Ono \\ Department of Astronomy and Geophysics, Tohoku University, Aramaki, Aoba, Sendai 980-8578, Japan
}

(Received September 24, 1997; Revised December 15, 1997; Accepted January 12, 1998)

\begin{abstract}
A new altimeter has been developed for the observation of the land shape of a planet from an orbiting spacecraft by transmitting RF pulses. The present system utilizes an RF sounder for observing the electron density profile of the topside ionosphere by selecting a transmission frequency $f$ higher than the local cutoff frequency of the ionosphere by adding upper and lower side band modulations with a frequency $p$; i.e. at frequencies $f+p$ and $f-p$. The phase difference of the propagating signals at the upper and lower side band frequencies gives a fine delay time with a range resolution of $100 \mathrm{~m}$ for $p=50 \mathrm{kHz}$ and $20 \mathrm{~m}$ for $p=250 \mathrm{kHz}$ with $f=9 \mathrm{MHz}$ corresponding to a resolution of $6^{\circ}$ of the phase detector of the system onboard the spacecraft. Using 600 Watts transmitting power applied to a $52 \mathrm{~m}$ tip-to-tip dipole antenna, the altitude of the spacecraft and the land shape can be measured while the spacecraft is in the range below $300 \mathrm{~km}$ altitude at the planet.
\end{abstract}

\section{Introduction}

A swept frequency radio wave sounder has been designed to be installed on the Planet-B spacecraft for the observation of the Martian topside ionosphere using pulsed radio waves in the frequency range from $20 \mathrm{kHz}$ to $5 \mathrm{MHz}$. In addition to this regular ionosphere sounding mode a function of altimeter has been installed extending frequency range to cover between 8 to $10 \mathrm{MHz}$. When the transmitting frequency is selected to be higher than the local cutoff frequency of the radio waves at the planetary ionosphere, the transmitted radio wave approaches the planetary surface. By measuring the propagation time of the transmitted pulse between the spacecraft and the planetary surface, we can detect the altitude of the spacecraft with respect to the sub-satellite point. A weight-saving system has been designed for the altimeter; i.e., the system of radio wave transmission and reception of the ionosphere sounder is utilized only by changing the transmitting frequency. The altimeter system can be applied to measure the land shape of Mars by detecting the variation of altitude with respect to the smoothly averaged spacecraft altitude.

The most popular and well established technique of the altimeter or the range shape sounder is the synthetic-aperture radar (Ulaby et al., 1981) using a higher frequency range than the S-band. In this context, the present system is quite unique and no previous example appears to be compatible with this technique. The uniqueness of the present altimeter lies in the following three points: (i) The radio sounder technique is applied only by changing the frequency to penetrate the ionosphere; therefore the frequency range is in the middle of the decameter waves. (ii) The altimeter uses coherent waves and, with the measurement of the pulse transmission

Copy right (C) The Society of Geomagnetism and Earth, Planetary and Space Sciences (SGEPSS); The Seismological Society of Japan; The Volcanological Society of Japan; The Geodetic Society of Japan; The Japanese Society for Planetary Sciences. time; the phase shift detection of the reflected waves provides sufficient resolution to determine the altitude with an error of $20 \mathrm{~m}$. (iii) Though decameter waves that are usually sensitive to ionospheric plasma density variation, are utilized, the ionosphere effect is completely eliminated by using two frequencies for the observation of the propagation time. Therefore the altimeter system is able to measure the altitude of a spacecraft or land shape without using any other data from different instruments.

Simple measurements of the pulse propagation time are limited in their resolution of the pulse-delay measuring process. The resolution of pulse-delay measurement, in the case of the RF sounder of the SPW on-board the Planet-B spacecraft is $25 \mu \mathrm{sec}$ which results in a resolution of $3.75 \mathrm{~km}$ for the determination of the altitude. To make a detailed observation of the land shape, therefore, a higher resolution is required. In the present altimeter, phase measurements of the signals are employed using modulation of the RF signal in the pulse to form lower and upper side band waves. By setting the RF to be $9 \mathrm{MHz}$, the developed system is able to determine the propagation range of the RF pulses between the spacecraft and the land shape with resolutions of $20 \mathrm{~m}$ and $100 \mathrm{~m}$, respectively for modulations frequencies of $250 \mathrm{kHz}$ and $50 \mathrm{kHz}$.

In this paper, the principle, instrumentation, and accuracy of the present altimeter, developed for observation of the land shape of Mars from the Planet-B spacecraft, are described.

\section{Principle}

When we select a radio wave frequency well above the maximum cut-off frequency of the ionosphere, the transmitted radio frequency waves can approach the planetary surface. By measuring the traveling time of the reflected waves, therefore, we can determine the relative altitude of the spacecraft with respect to the solid surface of the planet. The method of determining the propagation range $L$ using 
transmitted pulse depends on the frequency band width of the receiving system. In general propagation, pulse form $f\left(t-\frac{r}{c}\right)$ at point $r$, in a one-dimensional system, can be expressed by

$$
f\left(t-\frac{r}{c}\right)=\frac{1}{2 \pi} \int_{-\infty}^{\infty} F(\omega) \mathrm{e}^{i \omega\left(t-\frac{n(\omega) r}{c}\right)} d \omega,
$$

where $F(\omega)$ is the frequency spectra for the pulse in the complex regime measured at a given stationary point $r=0$; and $n(\omega)$ is the refractive index of the plasma media. When we consider a wide band case where integration of Eq. (1) is fully applicable, the pulse transmission is simply expressed with the condition

$$
t-\frac{n \cdot r}{c}=\text { const }
$$

that is,

$$
d t=\frac{n}{c} d r .
$$

The principle for the determination of altitude $L$ is then given by (see Jackson (1969) and reference therein)

$$
\int_{0}^{L} \frac{d r}{n(r, \omega)}=\frac{c \tau}{2}
$$

for the propagation time $\tau$ of the pulse, where $n(r, \omega)$ is the refractive index of the propagating wave at $r$ through the ionosphere. The refractive index $n(r, \omega)$ in a high frequency range well above the local-ionospheric plasma frequency $f_{p}$, can be expressed by

$$
n(r, \omega)=1-\frac{1}{2} \cdot \frac{\omega_{p}^{2}(r)}{\omega^{2}},
$$

where $\omega=2 \pi f$ for the transmitted frequency $f$ and $\omega_{p}=$ $2 \pi f_{p}$. The expression of Eq. (5) is rewritten as

$$
n=1-4.05 \times 10^{-5} \cdot \frac{N(r)}{f^{2}}
$$

for the frequency $f(\mathrm{MHz})$, and the electron density $N(r) / \mathrm{cc}$ at altitude $r$. When we select $f=9 \mathrm{MHz}$, for example, the second term of Eq. (6) is given within a range of less than 0.05 over the entire range of the propagation path.

When we apply a narrow frequency band signal receiving system, the detected pulse from $g(t, r)$ is written from Eq. (1), for approximately constant $n\left(\omega_{0}\right)$, as

$$
g(t, r)=\frac{1}{2 \pi} \cdot F\left(\omega_{0}\right) \exp \left\{i \omega_{0}\left[t-\int_{0}^{r} \frac{n\left(r, \omega_{0}\right)}{c} d r\right]\right\} \cdot \Delta \omega,
$$

where $\omega_{0}$ is the center frequency of the receiving channel and $\Delta \omega$ is the receiving frequency band width. The information of the propagating range $r$ is then expressed by the phase value of the detected signal that can be measured with reference to the original signal $\mathrm{e}^{i\left(\omega_{0} t+\Phi\right)}$, i.e., from the measured phase difference angle $\Phi_{\text {mes }}$, the range $r$ can be obtained from

$$
\int_{0}^{r} n\left(r, \omega_{0}\right) d r=\frac{c}{\omega_{0}} \cdot \Phi_{\mathrm{mes}}
$$

when we assume $n\left(\omega_{0}\right)$ nearly constant. Actually, we have utilized two different frequencies to measure the phase difference, i.e., for an angular frequency $\omega_{0}+2 \pi p$ the arrival signal is expressed as

$$
\begin{aligned}
S_{1}=a_{1} \cos [ & \left(\omega_{0}+2 \pi p\right) \\
& \left.\cdot\left\{t-\int_{0}^{r} \frac{n\left(r, \omega_{0}+2 \pi p\right)}{c} d r\right\}+\Phi_{1}\right],
\end{aligned}
$$

while the arrival signal is expressed as

$$
\begin{aligned}
S_{2}=a_{2} \cos [ & \left(\omega_{0}-2 \pi p\right) \\
& \left.\cdot\left\{t-\int_{0}^{r} \frac{n\left(r, \omega_{0}-2 \pi p\right)}{c} d r\right\}+\Phi_{2}\right]
\end{aligned}
$$

for the transmission of angular frequency, $\omega_{0}-2 \pi p$. To obtain the result signal $R$, the detected signal is treated as

$$
\begin{aligned}
& \underset{\tau}{R=1} \int_{t}^{t+\tau} S_{1} S_{2} d t \\
& =a_{1} a_{2} \cos \left[\frac{4 \pi p}{c} \int_{0}^{r}\left\{n\left(r, \omega_{0}\right)+\frac{\partial n}{\partial \omega} \omega_{0}\right\} d r\right. \\
& \left.+\left(\Phi_{2}-\Phi_{1}\right)\right],
\end{aligned}
$$

from which we can obtain $\Phi_{\text {mes }}$ as

$$
\begin{aligned}
\Phi_{\mathrm{mes}} & \stackrel{4 \pi p}{c} \int_{0}^{r}\left\{n\left(r, \omega_{0}\right)+\frac{\partial n}{\partial \omega} \omega_{0}\right\} d r \\
& +\left(\Phi_{2}-\Phi_{1}\right) .
\end{aligned}
$$

\section{Instrumentation}

In the altimeter onboard the Planet-B, wave transmission is designed to be made at the frequency $f-p$ and $f+p$ for $f=9 \mathrm{MHz}$ and two cases of $p$ at 50 and $250 \mathrm{kHz}$ with an approximate power level of 60 and 600 Watts. Two sets of $50 \mathrm{~m}$ tip-to-tip dipole antenna are installed; one is for the pulse signal transmission and the other is for the reception of the reflected pulse wave.

In Fig. 1, a block diagram of the altimeter system is given; the signal from the receiving antenna is fed to the pre-amplifier from the terminal $f_{R X}$ and the observed frequency is stepped up to a first-stage amplifier at the center frequency of $25 \mathrm{MHz}$. The local signals with frequencies

$$
f_{1}=f-p+f_{L} \quad \text { and } \quad f_{2}=f+p+f_{L}
$$

are generated for $f_{L}=25 \mathrm{MHz}$, in this case, using frequency synthesizers.

The present frequency synthesizer system is also utilized to generate the origin of the transmitting signal:

$$
\begin{aligned}
& a_{t \in} a_{1} \cos \left\{\left(\omega-2 \pi p+\omega_{L}\right) t+\varphi_{1}\right\} \\
& \left.\quad+a_{2} \cos \left\{\left(\omega+2 \pi p+\omega_{L}\right) t+\varphi_{2}\right\}\right] \\
& \cdot b \cos \left(\omega_{L} t+\varphi_{3}\right) .
\end{aligned}
$$

When the generated signal passes through a low pass filter, the signal given by Eq. (14) is further modified as

$$
\begin{aligned}
& L\left[a_{t} \nsucceq a_{1} b \cos \left\{(\omega-2 \pi p) t+\left(\varphi_{1}-\varphi_{3}\right)\right\}\right. \\
& \quad+a_{2} b \cos \left\{(\omega+2 \pi p) t+\left(\varphi_{2}-\varphi_{3}\right)\right\} .
\end{aligned}
$$




\section{Block Diagram of PWS/ALT}

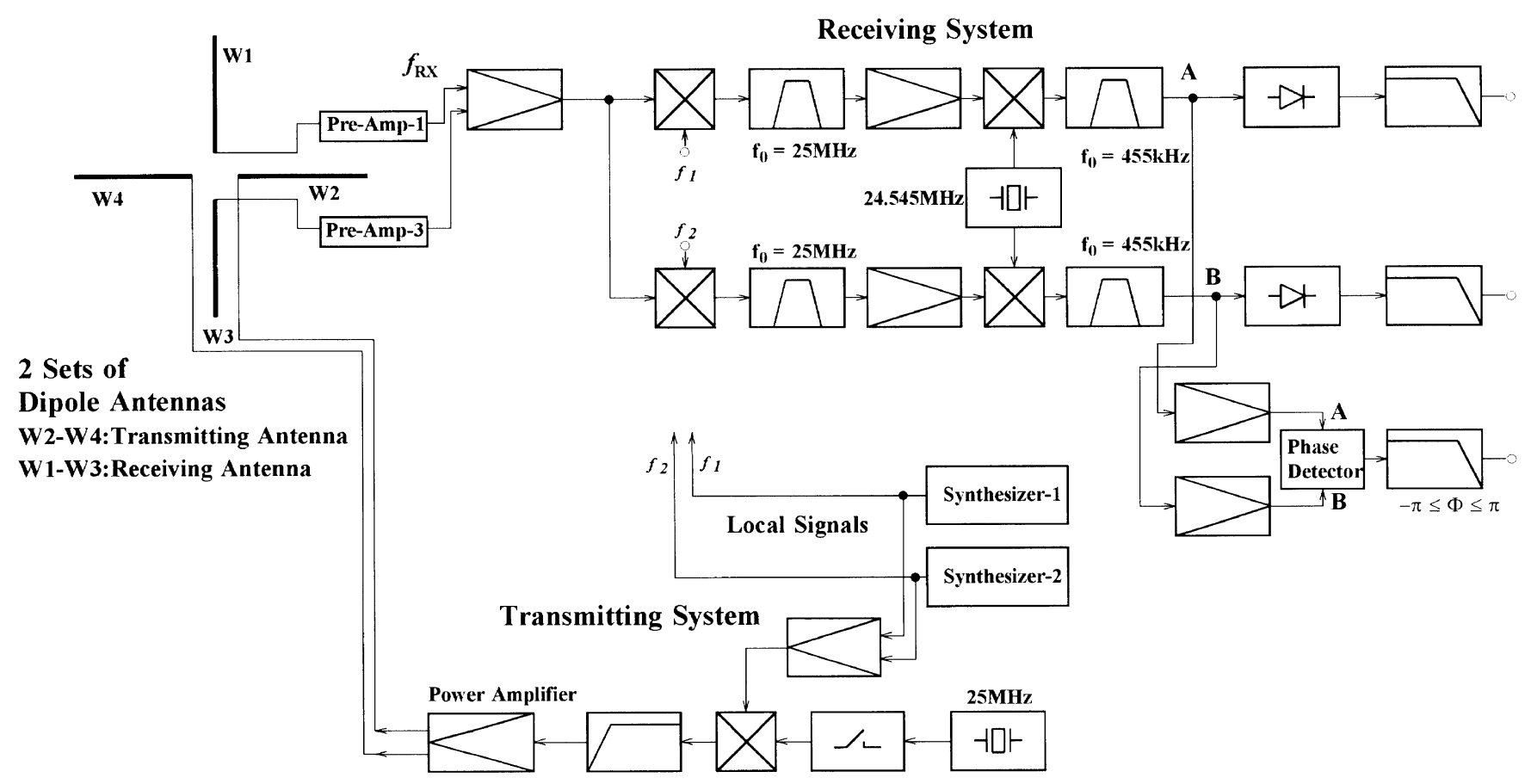

Fig. 1. Block diagram of an Altimeter system that consists of transmitting and receiving systems. The generated signal $a_{1} b \cos \left\{(\omega-2 \pi p) t+\left(\varphi_{1}-\varphi_{3}\right)\right\}+a_{2} b \cos \left\{(\omega+2 \pi p) t+\left(\varphi_{2}-\varphi_{3}\right)\right\}$ is fed to a power amplifier and transmitted from the transmitting antenna. The waves reflected by the planet's surface are picked up by the receiving antenna and fed to $f_{R X}$ point after passing through a pre-amplifier. The received signals are separated into two channels A and B through the process of frequency conversions into $25 \mathrm{MHz}$ signals. After amplification, the signals are further converted, keeping the phase relation between A and B to $455 \mathrm{kHz}$ signals, which are finally fed to a phase detector where the phase difference $4 \pi p\left(\frac{\Delta r}{c}\right)$ can finally be obtained.

This signal is amplified and fed to the power amplifier to be transmitted from the antenna.

By applying the two local signals (see Eq. (13)) during the reception phase which are given as

$$
a_{l 1}=A_{1} \cos \left\{\left(\omega-2 \pi p+\omega_{L}\right) t+\varphi_{l 1}\right\}
$$

and

$$
a_{l 2}=A_{2} \cos \left\{\left(\omega+2 \pi p+\omega_{L}\right) t+\varphi_{l 2}\right\}
$$

two signals are obtained which are both divided into the first converted stage as

$$
\begin{array}{r}
S_{\mp a_{r 1} A_{1} \cos \{} \omega_{L} t+\Phi_{1}-(\omega-2 \pi p) \\
\left.\cdot \int_{0}^{2 L} \frac{n(r, \omega-2 \pi p)}{c} d r\right\}
\end{array}
$$

and

$$
\begin{aligned}
S_{\bar{z} a_{r 2} A_{2} \cos }\left\{\omega_{L} t+\Phi_{2}-(\omega+2 \pi p)\right. \\
\left.\cdot \int_{0}^{2 L} \frac{n\left(r, \omega_{0}+2 \pi p\right)}{c} d r\right\},
\end{aligned}
$$

where $L$ is the distance between the spacecraft and the solid surface of the planet and $\Phi_{1}$ and $\Phi_{2}$ are the phase shifts in each signal path together with the instrumental phase shifts in the operating systems 1 and 2, respectively. The signals given by Eqs. (18) and (19) are amplified and converted to the intermediate frequency at the center frequency of $455 \mathrm{kHz}$. As shown in the block diagram (Fig. 1), two signals at $455 \mathrm{kHz}$ can be obtained at channels A and B. The phase detector set between $\mathrm{A}$ and $\mathrm{B}$ channels then gives the phase data between the signals given by Eqs. (18) and (19).

A first-order estimation of the altitude is only possible by measuring the delay time of the transmitted pulse; the time resolution for the measurement of pulse delay is designed here to be $25 \mu \mathrm{sec}$. The corresponding resolution of the altitude range is then limited to $3.75 \mathrm{~km}$. To obtain a fine resolution of the altitude within a given altitude range of $3.75 \mathrm{~km}$ accuracy, we must apply the phase detection system for two signals given by Eqs. (18) and (19).

Applying the operation given by Eq. (11), the resultant signal $R$ is obtained as

$$
\begin{aligned}
\operatorname{Rat}_{r 1} a_{r 2} A_{1} A_{2} \cos \{ & \frac{1}{c} \int_{0}^{2 L}[(\omega-2 \pi p) n(r, \omega-2 \pi p) \\
& -(\omega+2 \pi p) n(r, \omega+2 \pi p)] d r \\
& \left.+\left(\Phi_{2}^{\prime}-\Phi_{1}^{\prime}\right)\right\} .
\end{aligned}
$$

Because frequency conversion has been applied to the signals given by Eqs. (18) and (19), the phases of the corresponding channels are further shifted from $\Phi_{1}$ to $\Phi_{1}^{\prime}$ and $\Phi_{2}$ to $\Phi_{2}^{\prime}$. 
(a)

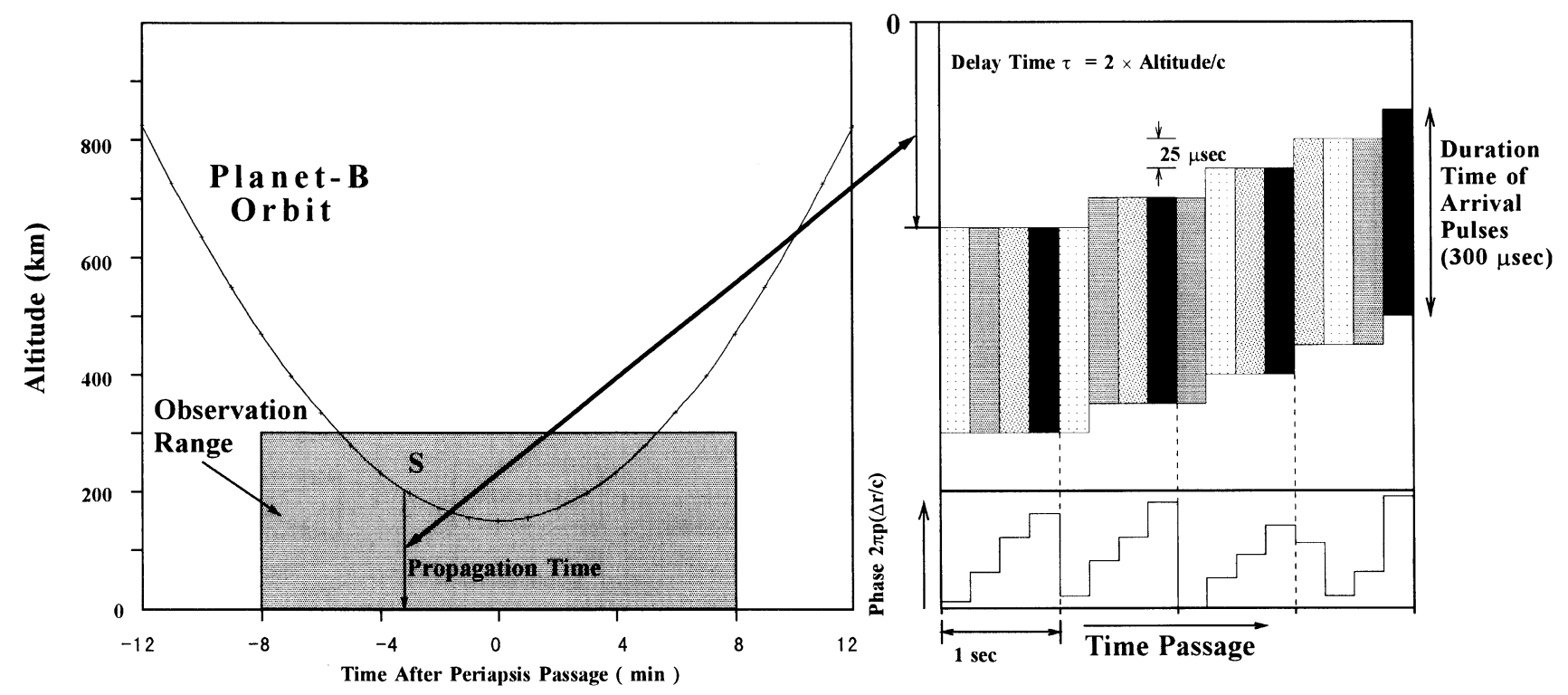

Fig. 2. Concept of the altimeter data at the time of observation onboard the spacecraft orbit around Mars. Panel (a) shows a planned orbit near the peri-aeron. At an example point on the orbit $\mathrm{S}$ in an altitude range lower than $300 \mathrm{~km}$, the altimeter data are indicated as in panel (b) where the pulse arrival time is illustrated at the top with the duration time of the arriving pulses, while a fine measurement in the range $\Delta r$ is made by phased measurement during the arriving period of the pulse ( $300 \mu \mathrm{sec}$ ). The phase data used to obtain the fine range $\Delta r$ versus time passage is given in the bottom part of panel (b); obtained phase data interpolate into a $3.75 \mathrm{~km}$ interval, corresponding to a time resolution of $25 \mu$ sec for measurements of the pulse arrival time.

Considering Eqs. (5) and (6), it then follows that

$$
\begin{aligned}
& (\omega-2 \pi p) \int_{0}^{2 L} \frac{n(r, \omega-2 \pi p)}{c} d r \\
& =\frac{2 L}{c}\left\{1-\frac{\alpha \omega_{p 0}^{2}}{\omega^{2}}\left(1+\frac{4 \pi p}{\omega}\right)\right\}(\omega-2 \pi p),
\end{aligned}
$$

where $\alpha$ is defined as a factor to satisfy

$$
\int_{0}^{2 L} 1.599 \times 10^{-3} N(r) d r=\frac{1}{2} \alpha \omega_{p 0}^{2} 2 L,
$$

i.e., $\alpha$ is an equivalent ratio of the ionosphere thickness to the entire path length with an assumed constant angular plasma frequency $\omega_{p 0}$, neglecting the term with the order of magnitude $(p / \omega)^{2}\left(\omega_{p}^{2} / \omega^{2}\right)$, Eq. (21) is further rewritten as

$$
\begin{aligned}
& (\omega-2 \pi p) \int_{0}^{2 L} \frac{n(r, \omega-2 \pi p)}{c} d r \\
& =\frac{2 L}{c}\left\{\omega\left(1-\frac{\alpha \omega_{p 0}^{2}}{2 \omega^{2}}\right)-2 \pi p\left(1+\frac{\alpha \omega_{p 0}^{2}}{2 \omega^{2}}\right)\right\} .
\end{aligned}
$$

In parallel to the operation of phase detection, we can detect $a_{r 1}$, and $a_{r 2}$ through the amplitude detection channel; then by making a calibration for a nonpropagation link at $L=0$, we can finally obtain the phase value from Eq. (20) by considering Eq. (23) for $\pm p$ as

$$
\Phi=4 \pi p\left(\frac{2 L}{c}\right)\left\{1+\frac{\alpha \omega_{p 0}^{2}}{\omega^{2}}\right\} .
$$

In actual data analyses, we can measure the phase variation $\Delta \Phi$ with respect to the variation of range $\Delta r$ that is defined as;

$$
\Delta r=2 L-m r_{0}
$$

for a given range $m r_{0}$, where $m$ is an integer and $r_{0}=75 \mathrm{~km}$; i.e.,

$$
\Delta \Phi=4 \pi p\left(\frac{\Delta r}{c}\right)\left\{1+\frac{\alpha \omega_{p 0}^{2}}{\omega^{2}}\right\} .
$$

In Fig. 2, the concept of expecting data from the altimeter is depicted in panel (b) relating to the planned orbit given in panel (a). When we select a point $S$ in the observation period of the altimeter, the pulses are observed with a time delay after transmission at $\tau=0$. Because of the resolution limit of $25 \mu \mathrm{sec}$ for measuring the timing of the arrival pulses, the apparent arrival time looks the same for several trials of the pulse propagation experiment, which made every $250 \mu \mathrm{sec}$. The measure of the pulse arrival time then jumps by $25 \mu \mathrm{sec}$ when the distance makes a corresponding variation. As is shown at the bottom of panel (b), the phase measurement of two-side band waves at $f+p$ and $f-p$ is made during the $300 \mu \mathrm{sec}$ time duration of the arriving pulse. Through this process high resolution data in terms of $4 \pi p(\Delta r / c)$, for $L=\left(m r_{0}+\Delta r\right) / 2$, are obtained.

\section{Accuracy-Discussion of Error}

Calibration for the phase measurement function has been made for the present instrument using a simulator to generate a pulse delay equivalent to that of the propagation. In Fig. 3, a block diagram of the calibration system is given. A phase delay equivalent to the propagation is produced by shifting the timing to the time-series data obtained by high speed sampling of the received wave form. The delay to this digitized time series data is controlled by an FIFO memory 


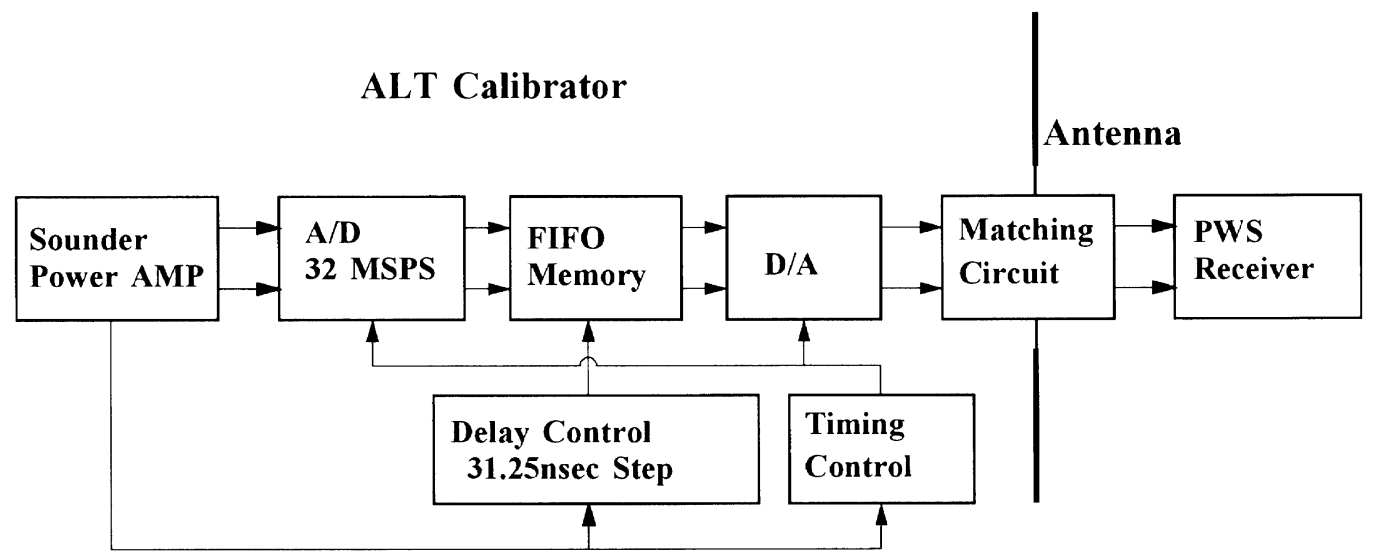

Transmission Timing Pulse

Fig. 3. Block diagram of the calibration system (ALT Calibrator) for phase delay. The output from the sounder power amplifier is fed to the A/D converter controling the power level. The time-series data obtained from the $\mathrm{A} / \mathrm{D}$ converter is shifted their timing from the reference timing of the pulse transmission by controlling the timing of the time-series data in the FIFO (First In First Out) memory with a time resolution of $0.03125 \mu$ sec. After making a delay equivalent to the time difference of the propagation, the digital time series data are fed to the D/A converter to form an actual case of an analog signal with actual $\mathrm{S} / \mathrm{N}$ ratio. The signal is then fed through the antenna's matching circuit to the receiver and the system for the measurement of the phase difference.

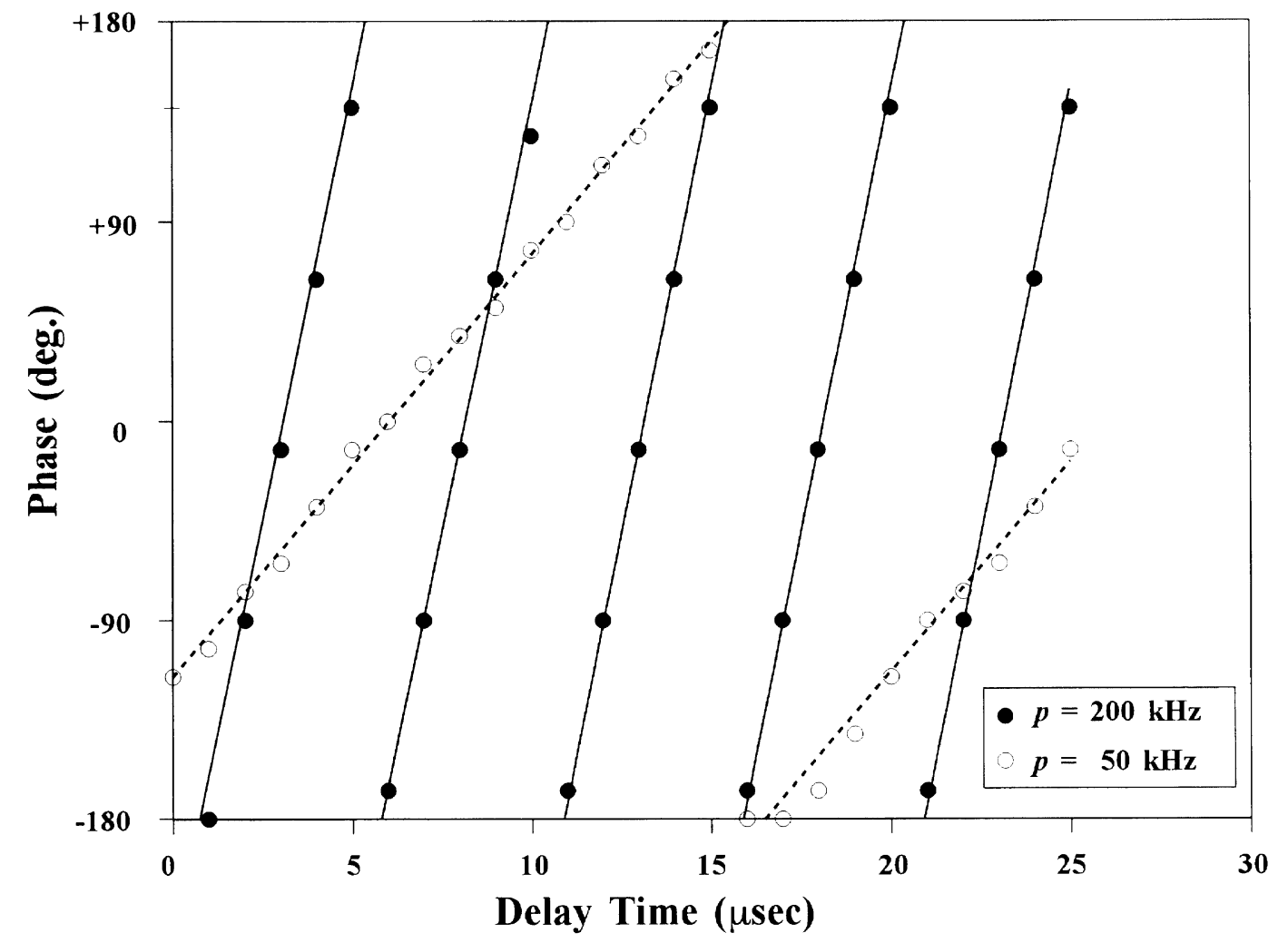

Fig. 4. Results of the measured phase (ordinate) versus the control time delay given in the abscissa. The measured phase angles are plotted for $p=200 \mathrm{kHz}$ (• symbols) and $p=50 \mathrm{kHz}$ ( $\circ$ symbols).

with a resolution of $31.25 \times 10^{-9} \mathrm{sec}$ (i.e., $0.03125 \mu \mathrm{sec}$ ). The digital data of the delay controlled time series are then converted to analog wave form again to feed the signal to $\mathrm{D} / \mathrm{A}$ converter to achieve a real wave form for an actual case of $\mathrm{S} / \mathrm{N}$ ratio. In Fig. 4, results of the measured phase angle $\Phi_{\text {mes }}$ (in ordinate) are indicated versus delay time. The re- sults verify that we can measure the phase difference with an error of $\pm 3^{\circ}$ for an actually possible $\mathrm{S} / \mathrm{N}$ ratio of the signal.

For an example case of $\alpha=1 / 5, f_{p 0}=3 \mathrm{MHz}$, and $f=9 \mathrm{MHz}$, the resolution limit $\left|\Delta \Phi_{\text {mes }}\right|$ is $6^{\circ}$; this limit results in $\Delta r=100 \mathrm{~m}$ for $p=50 \mathrm{kHz}$, while $\Delta r=20 \mathrm{~m}$ for $p=250 \mathrm{kHz}$, i.e., the estimate for the possible error of 


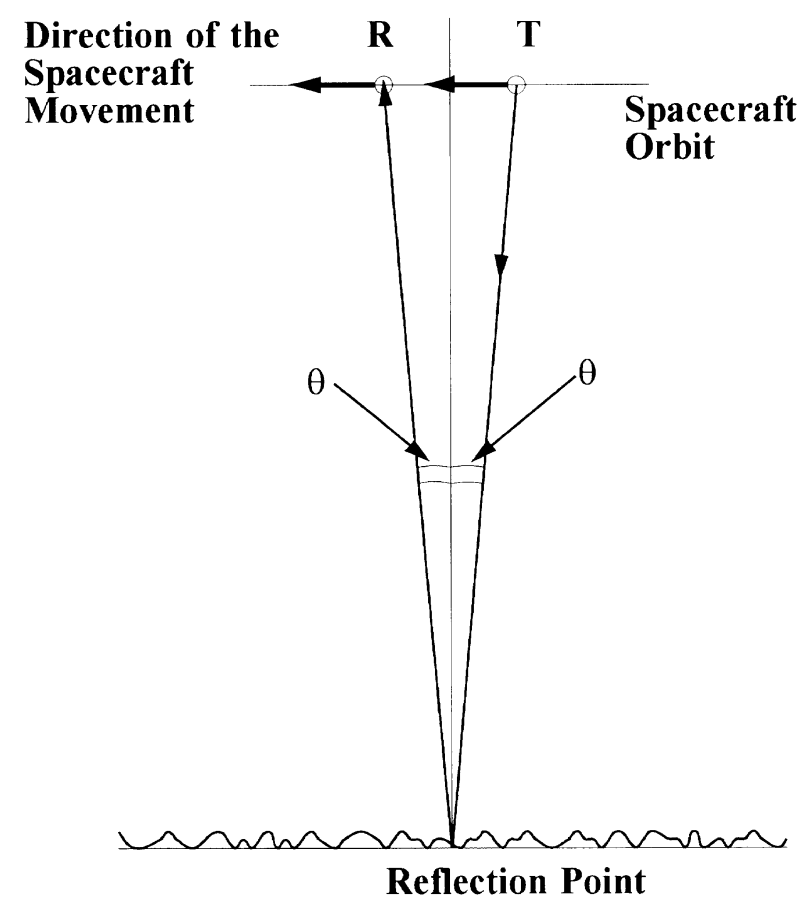

Fig. 5. Geometry of the spacecraft position and ray direction of the propagating waves.

a measured $\Delta \Phi$ value, is given by

$$
\delta(\Delta \Phi)=4 \pi p\left(\frac{r_{0}}{c}\right)\left\{\frac{\delta(\Delta r)}{r_{0}}+\alpha\left(\frac{\omega_{p 0}}{\omega}\right)^{2} \frac{\delta\left(\omega_{p 0}\right)}{\omega_{p 0}}\right\} .
$$

For $\Delta r \approx r_{0}=3.75 \mathrm{~km}$, corresponding $\delta\left(\omega_{p}\right)$ value to result the same error level of $\delta(\Delta r)=20 \mathrm{~m}$ is $0.72 \mathrm{MHz}$; therefore, the value means a plasma density variation of $40 \%$ in the ionosphere. Within the short period of time when the spacecraft passes over a special land shape, no such large density variation will take place. We can then neglect the variation effect of the ionospheric plasma density.

In this method, it is not necessary to consider the Doppler effect due to the relative motion of the spacecraft with respect to the reflection point. This is mainly because only the wave with the wave normal mostly in the perpendicular direction of the planetary average surface can be detected by the spacecraft. As has been given in Fig. 5, the deviation angle $\theta$ from the vertical direction is within the range of $3.3 \times 10^{-4}$ rad when we consider even five times the possible deviation distance of the spacecraft during the propagation time interval of the transmitted waves for $300 \mathrm{~km}$, assuming a spacecraft velocity of $8 \mathrm{~km} / \mathrm{sec}$. The Doppler frequency in this case is given by $\omega_{D}=2 k v \theta$, that gives the result, $\omega_{D}=8.8 \times 10^{-9} \omega$. We can therefore confirm that there is no disturbance from the Doppler effect in the present observation system. From the argument related to Fig. 5, we can also conclude that the illuminated area of measurement is around $100 \times 100 \mathrm{~m}$ when the land shape is approximately flat or convex, as in the cases of plains or mountains. The illuminated area may be increased for concave land shape.

\section{Conclusion}

Considering the chance to carry out an RF sounder experiment for the measurement of the topside ionosphere of Mars, a new altimeter has been developed utilizing the same principal facilities of the RF radio sounder. By selecting the frequency of the transmitted RF pulse to be higher than the local cutoff frequency of the planetary ionosphere, the reception of reflected pulse can be applied to the time of flight measurements of the spacecraft altitude with respect to the planetary surface. Because of the limitation of the time resolution for the measurement of the pulse arrival time, a simple application of time measurement of the pulse transmission gives a limit of $3.75 \mathrm{~km}$ for determination the spacecraft altitude, which corresponds to a time resolution of $25 \mu \mathrm{sec}$. To interpolate within this range limit for fine measurement, a phase detection system is added to the proper RF wave sounder technique.

The RF pulse signal with frequency $f$ is modulated with low frequency $p$ to make the upper side band at $f+p$ and the lower side band at $f-p$. The phase difference of these two side band signals is strictly related to the propagation range without significant effects of the ionospheric plasma.

The achieved instrumentation for the altimeter mode (ALTmode) of Planet-B PWS observation verifies that the altitude can be measured with a resolution of $100 \mathrm{~m}$ for $p=50 \mathrm{kHz}$ with $f=9 \mathrm{MHz}$ and a resolution of $20 \mathrm{~m}$ for $p=250 \mathrm{kHz}$ with $f=9 \mathrm{MHz}$, corresponding to a phase resolution angle of $6^{\circ}$ for the phase detector of the on-board system. It is also confirmed through simulation that the stable measurement of the altitude and land shape is possible within an altitude range below $300 \mathrm{~km}$, when a nominal 600 Watt power transmission is made, as has been achieved for the proper RF sounder of the Planet-B spacecraft.

Acknowledgments. The present study was carried out under the Planet-B project of ISAS. The authors are very grateful to all team members of the project.

\section{References}

Jackson, J., The reduction of topside ionograms to electron-density profiles, Proc. IEEE, 57, 960-976, 1969.

Ulaby, F. T., R. K. Moore, and A. K. Fung, Microwave remote sensing fundamentals and radiometry, Reading Mass., pp. 456, 1981.

H. Oya (e-mail: oya@stpp1.geophys.tohoku.ac.jp) and T. Ono (e-mail: ono@stpp1.geophys.tohoku.ac.jp) 